\title{
Evaluating the impact of maintenance policies associated to SLA contracts on the dependability of data centers electrical infrastructures
}

\author{
Avaliando o impacto de políticas de manutenção vinculadas a contratos de SLA na \\ dependabilidade das infraestruturas elétricas de data centers
}

Felipe F. de Lima Melo ${ }^{1 *}$,Gustavo R. de Almeida Callou ${ }^{1}$, João F. da Silva Júnior ${ }^{1}$

\begin{abstract}
Due to the growth of cloud computing, data center environment has grown in importance and in use. Data centers are responsible for maintaining and processing several critical-value applications. Therefore, data center infrastructures must be evaluated in order to improve the high availability and reliability demanded for such environments. This work adopts Stochastic Petri Nets (SPN) to evaluate the impact of maintenance policies on the data center dependability. The main goal is to analyze maintenance policies, associated to $S L A$ contracts, and to propose improvements. In order to accomplish this, an optimization strategy that uses Euclidean distance is adopted to indicate the most appropriate solution assuming conflicting requirements (e.g., cost and availability). To illustrate the applicability of the proposed models and approach, this work presents case studies comparing different SLA contracts and maintenance policies (preventive and corrective) applied on data center electrical infrastructures.
\end{abstract}

Keywords: Dependability - Maintenance - Stochastic Petri Nets - Data Center - SLA

Resumo: Devido ao crescimento da computação em nuvem, o ambiente de data center vem crescendo em importância e em utilização. Os data centers são responsáveis por manter e processar diversas aplicações de valor crítico e, assim, a análise da disponibilidade e confiabilidade de tais sistemas se torna de fundamental importância. Este trabalho adota as redes de Petri estocásticas (SPN) para avaliar o impacto das políticas de manutenção na dependabilidade de data centers. O intuito é fazer uma análise das políticas de manutenção normalmente empregadas vinculadas a contratos de $S L A$ e, assim, poder propor melhorias. Para se conseguir realizar tais análises, esse trabalho faz uso de uma estratégia de otimização que considera a distância euclidiana para indicar a solução mais adequada assumindo requisitos conflitantes (ex., custo e disponibilidade). Para ilustrar a aplicabilidade dos modelos e da abordagem proposta, estudos de caso foram conduzidos comparando diferentes contratos de SLA e políticas de manutenção (preventivas e corretivas) aplicadas a infraestruturas elétricas de data center.

Palavras-Chave: Disponibilidade - Manutenção - Redes de Petri Estocásticas - Data Center - SLA

${ }^{1}$ Departamento de Computação - Universidade Federal Rural Pernambuco (UFRPE) - Recife

*Corresponding author: felipe.Imelo@ufrpe.br

DOI: http://dx.doi.org/10.22456/2175-2745.88822 • Received: 11/12/2018 • Accepted: 15/10/2019

CC BY-NC-ND 4.0 - This work is licensed under a Creative Commons Attribution-NonCommercial-NoDerivatives 4.0 International License.

\section{Introdução}

Atividades relacionadas à manutenção de equipamentos industriais vêm sendo adotadas desde a revolução industrial, onde diferentes estratégias de manutenção podem ser empregadas com a finalidade de reduzir a indisponibilidade dos sistemas [1]. Tendo em vista o constante crescimento no nível de utilização dos ambientes que provêem suporte à computação em nuvem, tornam-se fundamentais estudos em relação a infraestrutura deste serviço para garantir a alta disponibilidade exigida. Dependendo da aplicação que esteja fazendo uso desse ambiente, uma falha do sistema pode gerar um prejuízo financeiro e, também, prejudicar a imagem da empresa. Sendo assim, espera-se que este ambiente esteja sempre operacional e sem ocorrência de falhas ou anomalia na sua infraestrutura.

Outro conceito importante é o de custo, que consiste no gasto relacionado a um bem ou serviço para produzir outros bens e serviços [2]. Neste trabalho, busca-se a redução dos custos, associados ao consumo de energia elétrica, através da adoção das melhores estratégias de manutenção. Porém, 
mesmo com a redução da infraestrutura, espera-se manter a oferta de serviços e capacidade de atender às necessidades do negócio [3]. Este trabalho leva em consideração o custo dos contratos de $S L A$, que contabiliza os custos de aquisição dos componentes que fazem parte da infraestrutura elétrica do data center, o custo de manutenção, o custo do time de manutenção e o custo do desgaste dos equipamentos.

Duas abordagens de manutenção distintas são utilizadas a fim de se evitar ou reduzir possíveis interrupções no funcionamento do sistema. A manutenção corretiva, é realizada quando algum dispositivo falhar; a segunda abordagem, a manutenção preventiva, visa evitar que um determinado dispositivo venha a falhar. Além disso, contratos de $S L A$, podem ser firmados para definir um conjunto de compromissos oferecido por uma empresa prestadora de serviço, possibilitando assim a escolha do melhor contrato levando em consideração o custo e a disponiblidade oferecida para quem vai contratar o serviço.

Este trabalho propõe uma metodologia que utiliza redes de Petri estocásticas (SPN) para avaliar o impacto das políticas de manutenção na dependabilidade das infraestruturas elétricas de data centers, levando em considera- ção contratos de SLA. Esse trabalho faz uso também da distância Euclidiana, para identificar, entre os resultados obtidos, o contrato que apresente o melhor custo-benefício em relação às métricas de interesse.

Este artigo está organizado da seguinte forma. A Seção II apresenta os trabalhos relacionados. A Seção III apresenta conceitos fundamentais sobre a infraestrutura de um data center. A Seção IV detalha os modelos básicos propostos. A Seção V explica a metodologia proposta, bem como des-creve os conceitos básicos sobre políticas de manutenção (preventiva e corretiva), contratos de $S L A$, disponibilidade e confiabilidade. A Seção VI ilustra a aplicabilidade da abordagem proposta através de um estudo de caso do mundo real e, por fim, a Seção VII conclui este trabalho e apresenta os futuros direcionamentos desta pesquisa.

\section{TRABALHOS RELACIONADOS}

Os autores em [4] apresentam uma avaliação referente as políticas de manutenção com diferentes SLAs. Uma modelagem híbrida é adotada para considerar as vantagens de diferentes redes de Petri estocásticas (SPN) e diagramas de bloco de confiabilidade (RBD) para avaliar infraestruturas de energia do data center, levando em consideração políticas de manutenção. No entanto, este trabalho focou somente em manutenção corretiva.

Silva et al. [5] apresentam uma metodologia para estimar métricas de impacto na sustentabilidade e confiabilidade, apoiada por um ambiente integrado conhecido como ASTRO. Essa ferramenta considera as vantagens dos diagramas de blocos de confiabilidade e das redes de Petri estocásticas para estimar o impacto da sustentabilidade com base na avaliação do ciclo de vida. Nada foi mencionado em relação a políticas de manutenção.
Os autores em [6] apresentam modelos de desempenho com a finalidade de avaliar os sistemas de computação em nuvem distribuídos em vários data centers, levando em consideração a ocorrência de desastres. Este trabalho apresenta uma abordagem para avaliar a capacidade de desempenho nos sistemas IaaS implantados em data centers geograficamente distribuídos. Modelos de dependabilidade foram propostos, mas não levaram em consideração manutenção preventiva e nem corretiva.

Os autores em [7] propõem modelos de dependabilidade que levam em consideração SLA. O foco dos autores foi somente na manutenção preventiva, cenários da manutenção corretiva não foram avaliados.

Said et al. [8] apresentam uma análise para quantificar os efeitos de múltiplas políticas de manutenção preventivas sobre a confiabilidade e disponibilidade de equipamentos móveis. Considera-se um sistema complexo e reparável, como um caminhão de transporte subterrâneo submetido a manutenção corretiva e vários tipos de manutenção preventiva (MP). Esse trabalho propôs modelos de dependabilidade e manutenção, mas o foco não foi em data centers.

Os autores em [9] apresentam uma avaliação de desempenho de um sistema, através de cadeias de Markov, manutenção preventiva e reparos mínimos. Esse trabalho não mencionou manutenção corretiva.

Em [10], os autores apresentam uma abordagem que quantifica a confiabilidade de redes elétricas, considerando a existência de equipamentos inteligentes capazes de isolar áreas com falhas, redirecionando a corrente elétrica de uma subestação vizinha para suprir a interrupção de energia. Os autores fazem uso de cadeia de Markov com recompensas para quantificar métricas relacionadas a disponibilidade. Manutenção não foi o foco dessa pequisa.

Um modelo de benchmarking para índices de confiabilidade é apresentado em [11]. O modelo encontra o componente de melhor desempenho entre o conjunto de componentes semelhantes com base nos conjuntos de entradas (porcentagem de alta-tensão (HT), alimentadores, porcentagem de cabos subterrâneos (UG), densidade de carga e vida útil restante dos transformadores) e saídas (SAIDI, SAIFI, ASAI).

[12] propõe uma abordagem geral de quantificação de sobrevivência que é aplicável a uma ampla gama de arquiteturas de sistemas e aplicativos. Essa abordagem proposta fornece informações úteis para melhor compreensão do estado estacionário do sistema e comportamento transitório sob várias condições adversas. Os autores fazem uso de uma modelagem utilizando cadeia de Markov. Os autores fazem uso da manutenção preventiva, porém nada foi mencionado em relação a manutenção corretiva.

A Tabela 1 ilustra os trabalhos relacionados, ressaltando as diferenças entre o foco de cada pesquisa. Conforme pode ser observado, poucos trabalhos abordam de forma integrada a modelagem de dependabilidade, da manutenção corretiva e preventiva com contratos $S L A$ para infraestruturas elétricas 
Table 1. Resumo comparativo dos trabalhos relacionados.

\begin{tabular}{ccrccc}
\hline Trabalhos Relacionados & Manutenção & SLA & Disponibilidade & Confiabilidade & Infraestrutura elétrica \\
\hline [Callou et al. 2010] & Corretiva & $\mathrm{X}$ & $\mathrm{X}$ & $\mathrm{X}$ & $\mathrm{X}$ \\
[Silva et al. 2013] & & $\mathrm{X}$ & $\mathrm{X}$ & $\mathrm{X}$ \\
[Silva et al. 2014] & & $\mathrm{X}$ & $\mathrm{X}$ & $\mathrm{X}$ \\
[Addabbo et al. 2015] & preventiva & $\mathrm{X}$ & $\mathrm{X}$ & $\mathrm{X}$ & \\
[Said and Taghipour 2016] & corretiva e preventiva & & $\mathrm{X}$ & $\mathrm{X}$ & \\
[Wang et al. 2017] & preventiva & & $\mathrm{X}$ & $\mathrm{X}$ & \\
[Diniz et al.] & & $\mathrm{X}$ & $\mathrm{X}$ & $\mathrm{X}$ \\
[Bichpuriya et al. 2011] & & $\mathrm{X}$ & $\mathrm{X}$ & $\mathrm{X}$ \\
[Liu and Trivedi 2006] & preventiva & & & & $\mathrm{X}$ \\
[Este Trabalho] & corretiva e preventiva & $\mathrm{X}$ & $\mathrm{X}$ & $\mathrm{X}$ & $\mathrm{C}$ \\
\hline
\end{tabular}

de data center. A diferença entre o presente trabalho para os trabalhos apresentados consiste na integração entre políticas de manutenção corretiva e preventiva, com contratos de SLAs para diferentes tipos de infraestruturas elétricas de data center. Vale ressaltar que também é realizada a modelagem de dependabilidade para avaliar tais infraestruturas.

\section{FUNDAMENTAÇÃO TEORICA}

Esta seção apresentada os conceitos de disponibilidade, confiabilidade, contratos de $S L A$ e políticas de manutenção.

\subsection{Políticas de Manutenção}

Define-se manutenção como sendo, um conjunto de ações desenvolvidas com o intuito de assegurar o bom funcionamento das máquinas e instalações. Os tipos de manutenção são as formas de encaminhar as intervenções nos instrumentos de produção, ou seja, nos equipamentos que compõem um determinado sistema [13]. Os principais tipos de manutenção são apresentados a seguir.

Manutenção preventiva, representa ações realizadas na tentativa de prevenir e reduzir a ocorrência de falhas ou queda no desempenho. Essa manutenção é realiza através da substituição de partes do sistema, obedecendo a um planejamento, baseado em períodos de tempo pré-estabelecidos, para determinado tipo de equipamento [14]. Vale ressaltar que este tipo de manutenção pode ser realizada em equipamentos operacionais, sendo assim, os equipamentos podem estar em estado ativo e sem apresentar falhas [15].

Manutenção corretiva realiza a correção de uma falha que ocorreu de maneira aleatória, ou seja, trata-se de uma correção da falha ou quando o sistema está apresentando um desempenho menor que o esperado. A manutenção corretiva implicar em altos custos, pois causa perda da produção além do custo de reparo do equipamento [16].

Manutenção preditiva é baseada nas condições do equipamento. Permitindo o acompanhamento do equipamento através de medições, acompanhamento de parâmetros ou condições de desempenho do equipamento [17]. Apresenta como objetivo o acompanhamento de paramentos, permitindo que o equipamento continue operacional por um maior período, sem apresentar nenhuma variação na sua disponibilidade, confiabilidade dos equipamentos [17].

Manutenção detectiva atua de forma a detectar falhas ocultas nos equipamentos que não podem ser percebidas pela equipe de manutenção e operação. Ela é aplicada em sistemas de proteção, comando e controle em que a ocorrência de falha tem que ser a mínima possível [18]. Apresenta a finalidade de atender a toda uma gama de equipamentos e modos de falhas que não se enquadram em qualquer uma das políticas tradicionais. Sendo assim, o diagnóstico é obtido de forma direta a partir do processamento das informações colhidas, a identificação de falhas ocultas é primordial para garantir a confiabilidade do sistema [19].

\subsection{Dependabilidade}

A dependabilidade denota a capacidade que um sistema tem de oferecer um serviço de forma confiável. Esse trabalho foca nas duas principais medidas de dependabilidade: disponibilidade e confiabilidade. A disponibilidade de um sistema é definida como a fração do tempo que o sistema está disponível para atender os pedidos dos usuários [20]. O tempo durante o qual o sistema não está disponível é chamado de tempo de inatividade.

Para avaliar a disponibilidade do sistema são considerados: tempo médio para a falha (Mean Time to Failure - MTTF), trata-se do tempo médio para a ocorrência de uma falha; tempo médio para reparo (Mean Time to Repair - MTTR), trata-se do tempo médio que se leva para executar um reparo após a ocorrência da falha. A disponibilidade (A) de estado estacionário pode ser calculada pela Equação 1:

$$
A=\frac{M T T F}{M T T F+M T T R}
$$

\subsection{Confiabilidade}

A confiabilidade de um sistema é a probabilidade (P) de que esse sistema execute a sua função, de modo satisfatório, sem a ocorrência de defeitos, por um determinado período de tempo (T) [21]. A Equação 2 é utilizada para se computar a 
confiabilidade.

$$
R_{(t)}=P\{T>t\}, t \geq 0=\int_{t}^{\infty} f(x) d x
$$

Onde $\mathrm{T}$ é o período de tempo para se encontrar a falha, $\mathrm{f}(\mathrm{x})$ representa a função densidade de probabilidade de falha e té o período de tempo considerado.

\subsection{SLA}

Service Level Agreement (SLA) é um acordo de nível de serviço, e resulta em um documento que define um conjunto de compromissos entre uma empresa prestadora de serviços e um cliente [22]. Sendo assim, através deste documento o cliente tem acesso aos serviços oferecidos pela empresa. Este documento descreve os serviços a serem obtidos e as taxas a serem alcançadas para o cumprimento de todos os compromissos acordados [23].

Um contrato de $S L A$ pode ser definido como sendo um acordo entre o serviço de suporte e o usuário que quantifica o serviço mínimo aceitável [24]. Os contratos de SLA são particularmente úteis no processamento crítico podendo ser documentos bem complexos e longos ou podem ser simples. De toda forma, SLAs são indispensáveis para proporcionar bons relacionamentos de serviço entre o vendedor e o cliente [24].

Um SLA também especifica as medidas a serem tomadas em caso de desvio e falta de atendimento ao serviço contratado. As afirmações do provedor de serviços são baseadas em uma definição detalhada dos parâmetros de serviço [25].

Os contratos de SLAs foram adotados, neste trabalho, para proporcionar a elaboração de cenários distintos entre si. Contudo, cada um apresenta suas próprias características, definindo, por exemplo, um conjunto de compromissos que devem ser atendidos.

\section{Modelos}

A Figura 1 ilustra o modelo base de dependabilidade em SPN para representar um sistema com um único componente. Neste modelo, o equipamento pode adotar dois possíveis estados: em funcionamento ou falho. Como pode ser observado, existem duas transições que representam respectivamente o tempo médio para a falha e reparo deste equipamento. Neste caso, a transição MTTF representa o tempo médio para o equipamento UPS falhar, já a transição MTTR representa o tempo médio para reparo do equipamento. Um token no lugar UPS_ON ou UPS_OFF representa, respectivamente, o funcionamento ou falha de um equipamento, no caso, de um UPS. A disponibilidade pode ser computada ao calcular a probabilidade do sistema se encontrar em funcionamente, ou seja, probabilidade de se ter token no lugar UPS_ON $\left(P\left\{\# U P S \_O N>0\right\}\right)$. A confiabilidade é computada usando a mesma métrica sem permitir, no entanto, que sejam realizados reparos no sistema.

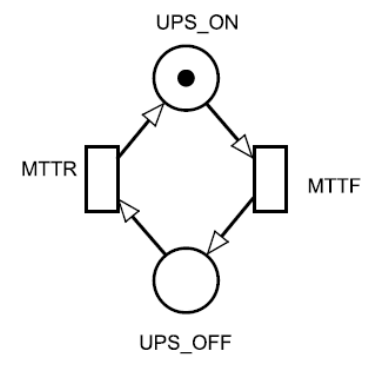

Figure 1. Modelo Base

\subsection{Modelo Manutenção Corretiva}

A Figura 2 ilustra o modelo criado para representar o modelo base, onde um time de manutenção corretiva é alocado para realizar a manutenção. Um token no lugar repararUPS representa o momento em que o equipamento se encontra em falha e foi submetido a uma manutenção corretiva. Entretanto, para que este lugar seja alcançado, faz-se necessário que uma equipe de manutenção esteja disponível (um token no lugar equipeManutencao). Pode-se observar que existem três transições no modelo: MTTR, MTTF e acionarManutencao. As duas primeiras foram explicadas anteriormente no modelo base. A transição imediata acionarManutencaoUPS é disparada assim que o dispositivo falha e a equipe de manutenção está disponível para iniciar o reparo. Vale ressaltar que um único lugar é adotado para o time de manutenção. Sendo assim, se estiverem presentes vários equipamentos no sistema, a equipe de manutenção só vai poder ser acionada para fazer um único reparo por vez.

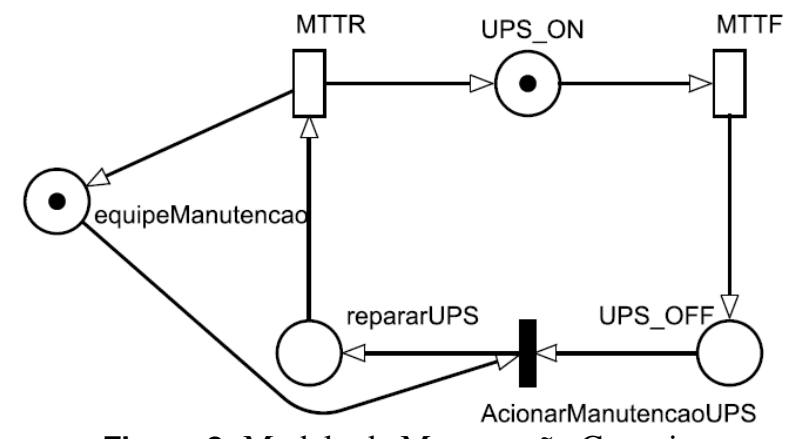

Figure 2. Modelo da Manutenção Corretiva

O modelo de manutenção corretiva contempla ainda a modelagem de um clock (ver Figura 3) para representar o passar do tempo durante o período de avaliação, por exemplo, 5 anos. Um token no lugar primeiroAno, segundoAno, terceiroAno, quartoAno ou quintoAno, representa a simulação do tempo decorrido. As transições estocásticas apresentadas nesse modelo possuem um tempo de um ano (8760 horas). Este clock é utilizado para representar o desgaste que o equipamento terá, ao passar dos anos, onde tem-se uma degradação de $10 \%, 20 \%, 30 \%, 40 \%$ e $50 \%$, respectivamente no tempo entre falhas. Em outras palavras, com o passar do tempo, os equipamentos tendem a falhar com uma maior frequência. Conforme ilustra a Figura 3, o to- 
ken estará no lugar correspondente a simulação do tempo decorrido. Dependendo de onde o token esteja, a degradação pode aumentar até atingir o máximo de 50\%. Esse comportamento é representado pela fórmula do delay que é associada às transições de falha do modelo. Por exemplo, a expressão associada a transição MTTF é MTTF_UPS - (MTTF_UPS X 0.1 X \#primeiroAno) - (MTTF_UPS X 0.2 X \#segundoAno) (MTTF_UPS X 0.3 X \#terceiroAno) - (MTTF_UPS X 0.4 X \#quartoAno) - (MTTF_UPS X 0.5 X \#quintoAno). Pode-se observar que se o clock estiver no lugar primeiroAno, o MTTF do UPS será reduzido em $10 \%$.

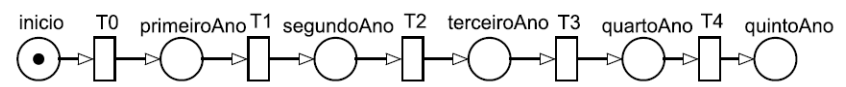

Figure 3. clock Modelo Manutenção Corretiva

\subsection{Modelo Manutenção Preventiva}

A Figura 4 representa o modelo base de manutenção corretiva, acrescido da possibilidade da realização de manutenção preventiva. Neste modelo existe a adição de um novo lugar, REPARAR_UPS_PREV, para representar o momento da ocorrência da manutenção preventiva. A transição INICIAR_REPARO_UPS simula a periodicidade para a realização da manutenção preventiva, por exemplo, 8760 horas representa um ano. A transição TERMINAR_REPARO_UPS representa o tempo necessário para a realização da manutenção preventiva no dispositivo, 4 horas, por exemplo. A grande diferença da manutenção preventiva para a corretiva é que o MTTF do dispositivo na manutenção preventiva é reparado para o valor do equipamento novo.

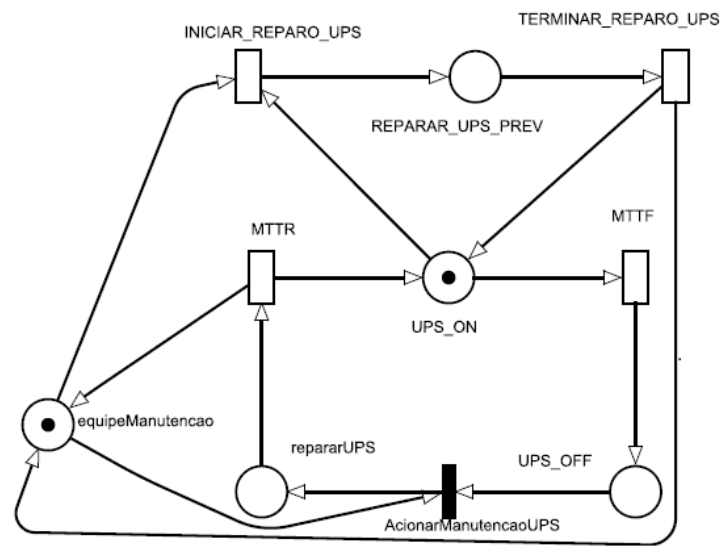

Figure 4. Modelo Manutenção Preventiva

A Figura 5 representa o clock da manutenção preventiva. Observa-se a existência das transições imediatas T6, T7, T8, T9 e T10 que representam a volta ao estado inicial, ou seja, quando ocorrer uma manutenção preventiva a degradação passa a ser zero (0), indicando que o dispositivo volta ao estado de novo. As transições T6, T7, T8, T9 e T10 serão ativadas caso a condição de guarda seja satisfeita. Para o exemplo da Figura 6, todas as transições imediatas do modelo da Figura 5 têm a seguinte expressão de guarda $\left(\left(\# R E P A R A R \_U P S \_P R E V>0\right) O R\left(\# R E P A R A R_{-} U P S \_P R E V 2\right.\right.$ $>0)$ ).

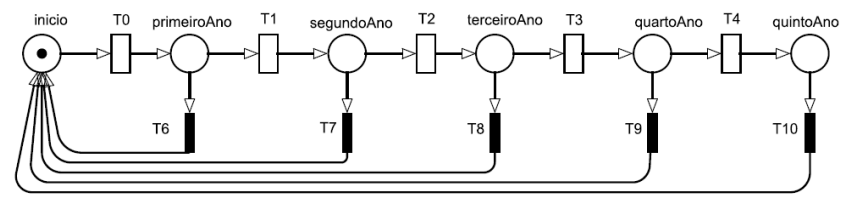

Figure 5. clock Modelo Manutenção Preventiva

Vale salientar que se temos dois dispositivos redundantes, só faz sentido realizar a manutenção preventiva em cada um dos equipamentos por vez. Dessa forma, a Figura 6 modela dois dispositivos UPS redundantes, com o acréscimo de um artificio para não permitir que os dispositivos UPS_ON e UPS_ON2 entrem em manutenção preventiva ao mesmo tempo. Esse artifício, trata-se de uma ligação entre UPS_ON e a transição INICIAR_REPARO_UPS2, e a ligação entre UPS_ON2 e a transição INICIAR_REPARO_UPS.

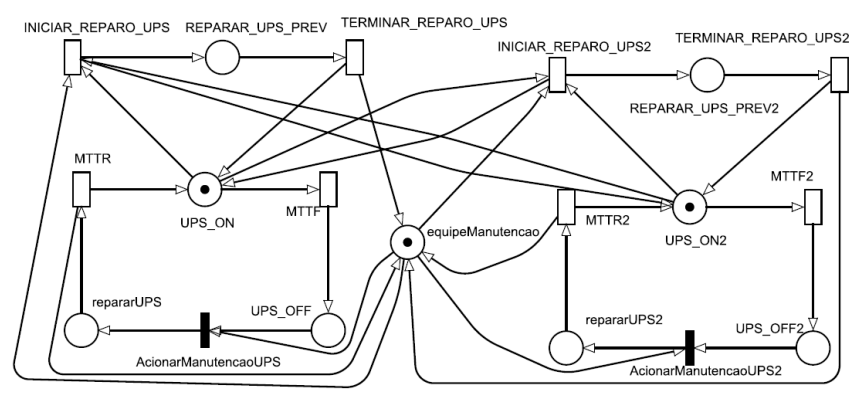

Figure 6. Modelo Manutenção Preventiva Modificado

\section{Metodologia}

Esta seção apresenta, a metodologia utilizada para avaliar a dependabilidade de arquiteturas de data centers levando em consideração políticas de manutenção vinculados a contratos de SLA. A Figura 7 mostra uma visão geral da metodologia que está sendo utilizada neste trabalho.

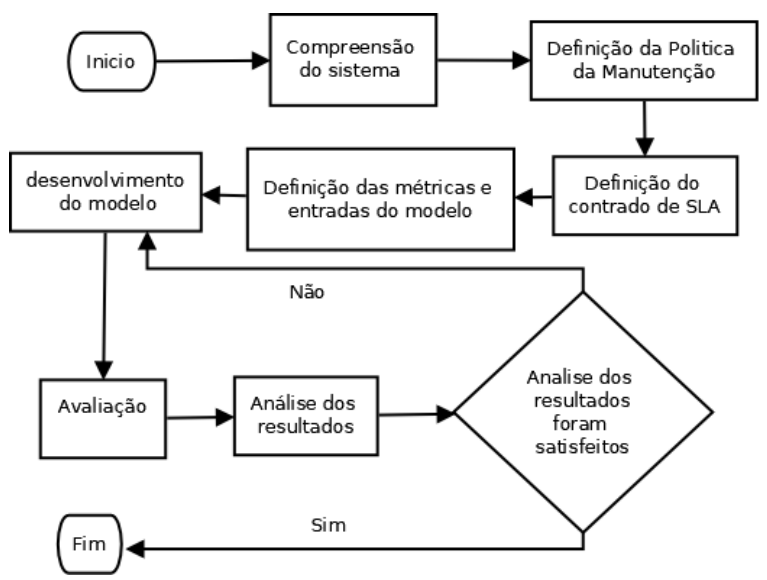

Figure 7. Metodologia adotada 
A primeira etapa da metodologia proposta corresponde à compreensão do sistema, onde é primordial entender o funcionamento do sistema real e, a partir deste, ser capaz de representá-lo por meio de modelos.

Na segunda fase são definidas as políticas de manutenção que vão ser adotadas. Por exemplo, pode-se adotar somente manutenção corretiva ou manutenção preventiva e corretiva. Na manutenção corretiva são levantados todos os parâmetros e características deste tipo de manutenção, tais como: MTTF e MTTR de cada equipamento. Assumindo a manutenção preventiva e corretiva, considera-se todos os aspectos citados para a manutenção corretiva com o acréscimo de parâmetros como a frequência em que serão realizadas as manutenções preventivas e o tempo que levam para serem realizadas.

Na terceira fase são definidos os contratos de SLA para cada tipo de manutenção (corretiva e preventiva/corretiva), onde são apontadas as condições necessárias para cada nível do contrato. Os contratos de SLAs para a manutenção corretiva considera as métricas: (i) o custo gasto com equipamento; (ii) o custo da manutenção corretiva; (iii) o custo com o time de manutenção. Para a manutenção preventiva, além dos custos citados, deve-se considerar também o custo extra da realização de uma manutenção periódica. Tendo em vista as métricas apresentadas, podem ser criados os seguintes contratos: SLA 1, SLA 2, SLA 3 e SLA para a manutenção corretiva com o tempo de reparo de $120 \mathrm{~h}, 48 \mathrm{~h}, 24 \mathrm{~h}$ e $8 \mathrm{~h}$, respectivamente. Já para a manutenção preventiva, além dos tempos utilizados, faz-se uso de um tempo extra, que corresponde a frequência da manutenção preventiva, podendo ser realizada a cada, dois anos (17520 horas), um ano e meio (13140 horas), 1 anos (8760 horas) e 6 meses (4380), por exemplo. Vale ressaltar que na Seção 6 cada custo citado e contratos criados são explicados de forma detalhada.

A quarta fase está relacionada com a definição das métricas e dados de entrada para o modelo. Os dados de entrada podem ser obtidos tanto experimentalmente como a partir dos dados fornecidos por manuais de fabricantes. Os dados de entrada correspondem ao MTTF, MTTR, quantidade de equipes de manutenção e ao custo associado a cada contrato SLA. Vale ressaltar que essa pesquisa foca em disponibilidade, confiabilidade e custo (aquisição e manutenção).

Uma vez definida a política de manutenção, segue-se com a criação do modelo de dependabilidade com a política de manutenção escolhida. Nessa fase é necessário escolher a ferramenta de modelagem (ex. TimeNet [26]), como também qual tipo de modelo será utilizado para representar o ambiente real. Por exemplo, pode-se adotar o formalismo de redes de Petri estocástica para representar a dependabilidade do sistema modelado que leva em consideração as políticas de manutenção estabelecidas.

Uma vez criado o modelo, deve-se partir para a fase de avaliação. Nesta etapa são realizadas diversas verificações quanto a corretude do modelo proposto para representar o sistema real. A verificação consiste em executar o modelo proposto e verificar se o mesmo se comporta corretamente, e com resultados consistentes.

Após a verificação do modelo é feita uma análise dos resultados obtidos a partir dos modelos e, assim, se consegue identificar e propor melhorias no sistema de data center modelado. Através dos modelos, diferentes cenários podem ser analisados com o objetivo de se melhorar a disponibilidade, por exemplo, ao se identificar a melhor política de manutenção em conjunto com o melhor contrato $S L A$. Vale salientar que esse trabalho faz uso da distância Euclidiana para auxiliar na identificação dos cenários analisados mais interessantes levando em consideração métricas conflitantes como custo e disponibilidade.

Por fim, caso a análise dos resultados obtidos não sejam satisfatória volta-se para a fase de desenvolvimento do modelo e ajustes são realizados a fim de que o modelo possa prover resultados válidos para o sistema em avaliação.

\section{Estudos de Caso}

Esta seção apresenta dois estudos de caso. O primeiro estudo tem como objetivo realizar uma comparação entre a manutenção corretiva e preventiva, a fim de, avaliar o impacto da manutenção na confiabilidade do sistema. $\mathrm{O}$ segundo estudo objetiva analisar o impacto das diferentes políticas de manutenção na disponibilidade das arquiteturas de data centers.

\subsection{Arquiteturas}

A Figura 8 apresenta as cinco arquiteturas das infraestruturas elétricas de data center adotadas nesses estudos [27]. Essas arquiteturas são compostas por: fontes de alimentação sem interrupção (UPS), transformador (SDT), chaveador de transferência estática (STS), quadro de energia (subpanel) e réguas de energia elétrica (powerstrip). A arquitetura base (A1) não tem nenhuma redundância, sendo composta por UPS, Subpanel, SDT e Powerstrip. Na arquitetura A2, adiciona-se um UPS de backup e um chaveador STS. Redundâncias são adiconadas até se ter toda a arquitetura base totalmente replicada (arquitetura A5). A Tabela 2 apresenta os MTTFs, MTTRs e custo de aquisição [27] dos equipamentos utilizados.

Table 2. MTTFs, MTTRs e custo de aquisição dos dispositivos

\begin{tabular}{lccc}
\hline Dispositivo & MTTF(h) & MTTR(h) & Custo de Aquisição \\
\hline UPS & 250000 & 8 & $\mathrm{R} \$ 21.553,29$ \\
STS & 24038462 & 6 & $\mathrm{R} \$ 2.282,28$ \\
SDT & 1412908 & 156,01 & $\mathrm{R} \$ 5.347,44$ \\
Subpainel & 1520000 & 2,4 & $\mathrm{R} \$ 208,61$ \\
Power Strip & 11511175,62 & 3,8 & $\mathrm{R} \$ 1.817,58$ \\
\hline
\end{tabular}

\subsection{Modelos}

Para todas as arquiteturas (A1, .., A5), foram analisados a disponibilidade e confiabilidade dos equipamentos quando esta é submetida a manutenção corretiva e preventiva. Sendo assim, o objetivo deste estudo de caso é realizar uma compara- 


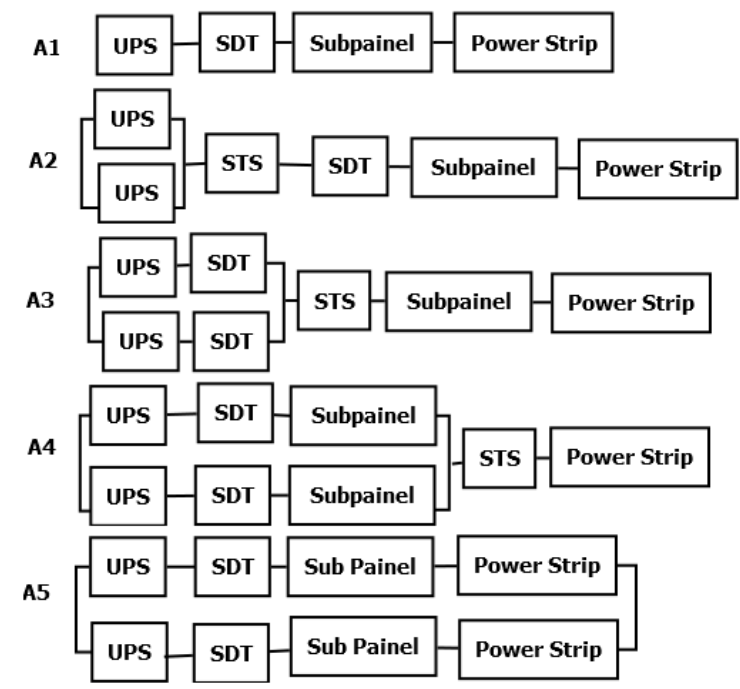

Figure 8. Arquiteturas

ção entre tais abordagens vinculadas a diferentes contratos de $S L A$ e identificar a que tem melhor impacto positivo na dependabilidade do sistema data center.

A Figura 9 apresenta o modelo SPN proposto para representar a disponibilidade obtida com a manutenção corretiva da arquitetura A1. Neste modelo, o equipamento só é reparado caso um dos equipamentos falhe e uma equipe de manutenção esteja disponível para ser alocada para fazer o reparo. A disponibilidade e/ou confiabilidade dessa manutenção é computada através da equação:A=(\#UPS_ON = 1) AND (\#SDT_ON =1) AND (\#Subpainel_ON = 1) AND (\#PowerStrip_ON = 1). O leitor deve recordar que para o cálculo da confiabilidade, nenhum equipamento pode ser reparado.

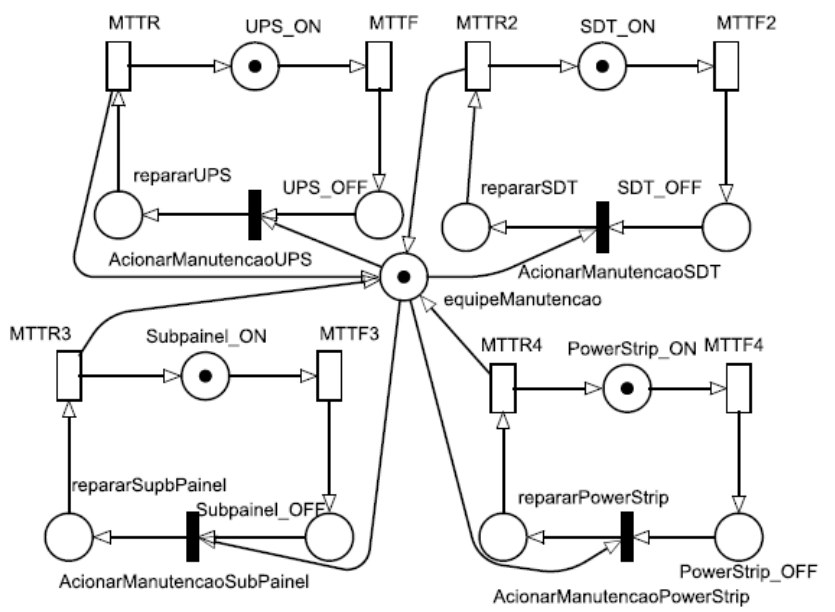

Figure 9. Modelo Arquitetura A1 Manutenção Corretiva

A Figura 10 apresenta o modelo SPN proposto para computar o impacto da manutenção preventiva na dependabilidade da arquitetura A2, observa-se que existe redundância do equipamento UPS. Neste modelo, o equipamento é reparado em caso de falha (manutenção corretiva), ou o equipamento pode ser submetido à manutenção preventiva (somente o UPS). A disponibilidade e/ou confiabilidade é computada através da equação: ((\#UPS_ON = 1) OR (\#UPS_ON2 = 1)) AND (\#SDT_ON =1 ) AND (\#Subpainel_ON = 1) AND (\#PowerStrip_ON = 1) AND (\#STS_ON = 1). Para a manutenção preventiva, a arquitetura A1 não foi analisada, visto que para melhorar a disponibilidade do serviço só faz sentido realizar a manutenção preventiva em equipamentos redundantes, ou seja, das arquiteturas A2 a A5.

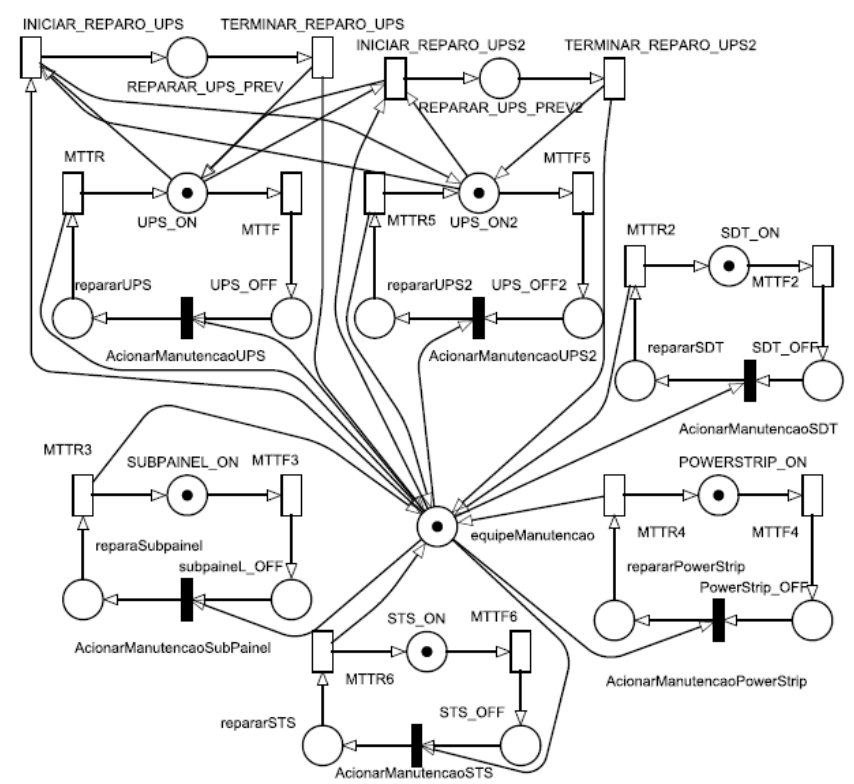

Figure 10. Modelo Arquitetura A2 Manutenção Preventiva

Vale ressaltar que para efeitos de simplificação da representação, os modelos das Figuras 9 e 10 foram apresentados sem os seus respectivos clocks, previamente descritos (Seção 4). Destaca-se ainda que todas as transições temporizadas têm o tempo definido de acordo com o clock, conforme mostra a expressão associada a cada transição $M T T F \_-\left(M T T F \_\times\right.$ $0.1 \times$ \#primeiroAno $)-($ MTTF $X \times 0.2 \times$ \#segundoAno $)-$ $($ MTTF $X \times 0.3 \times \#$ terceiroAno $)-($ MTTF_X $\times 0.4 \times$ \#quartoAno $)-($ MTTF $X \times 0.5 \times \#$ quintoAno $)$, onde $\mathrm{X}$ representa cada um dos equipamentos.

\subsection{Estudo de Caso I: Confiabilidade}

Esta seção tem como objetivo realizar uma comparação entre a manutenção corretiva e preventiva, a fim de avaliar o impacto de tais manutenções na confiabilidade do sistema. Vale ressaltar que os MTTFs e MTTRs utilizados se encontram representados na Tabela 2.

A Tabela 3 apresenta os resultados da confiabilidade para o período de 1 a 5 anos. É possível perceber, claramente, que houve uma diminuição considerável na confiabilidade do sistema ao passar do tempo, conforme esperado. Essa diminuição aconteceu em ambas as manutenções, ou seja, a confiabilidade tanto na manutenção corretiva quanto na manutenção preventiva tendeu a cair com o passar dos anos. 
Entretanto, os resultados obtidos para a confiabilidade do sistema utilizando a manutenção corretiva e para a preventiva foram os mesmos. Isso ocorreu devido ao fato de que na avaliação da confiabilidade não é permitida o reparo no sistema.

Table 3. Confiabilidade

\begin{tabular}{cccccc}
\hline Arquiteturas & 1 ano & 2 ano & 3 ano & 4 ano & 5 ano \\
\hline A1 & 0,95060480 & 0,89749760 & 0,84056620 & 0,78090042 & 0,72041516 \\
A2 & 0,98489448 & 0,96507785 & 0,93961232 & 0,90819486 & 0,87141008 \\
A3 & 0,99089306 & 0,97660622 & 0,95602442 & 0,92860769 & 0,89477094 \\
A4 & 0,99643358 & 0,98717674 & 0,97094742 & 0,94699544 & 0,91560154 \\
A5 & 0,99754814 & 0,98937846 & 0,97418525 & 0,95117640 & 0,92059162 \\
\hline
\end{tabular}

Analisando a Tabela 3 é possível observar que a arquitetura A5 obteve o melhor resultado da confiabilidade, com 92,06\% no quinto ano. Conforme esperado, mesmo com o passar dos anos, a arquitetura A5 ainda manteve a maior confiabilidade. Pode-se perceber que a confiabilidade do sistema, tende a cair ao passar dos anos, saindo de uma confiabilidade de $99,75 \%$ para uma de $92,06 \%$ para a arquitetura A5.

Foi avaliado, ainda, a confiabilidade do sistema com a utilização de uma, duas e três equipes de manutenção. Essa variação na quantidade de equipes de manutenção disponíveis foi realizada para verificar o impacto do número de equipes de manutenção na confiabilidade do sistema. Observou-se que a confiabilidade permaneceu, praticamente, a mesma ao passar dos anos, não sofrendo nenhuma alteração significativa, tanto para a manutenção corretiva quanto para a manutenção preventiva. Dessa forma, esses resultados não foram apresentados.

\subsection{Estudo de Caso II: SLA, disponibilidade e Distân- cia Euclidiana}

Essa seção apresenta um estudo de caso que tem o objetivo de analisar o impacto das diferentes políticas de manutenção, vinculadas a contratos de $S L A$, na disponibilidade das arquiteturas de data centers. Vale salientar que também analisamos o impacto da quantidade de equipes de manutenção nos contratos de SLA. Os resultados da disponibilidade alcançada por cada contrato são apresentados percentualmente e em número de noves (9's), calculados por meio da fórmula $(-\lg [1-A / 100])$, sendo A a disponibilidade. Considerando ambas as métricas de custo e downtime, foi utilizada a distância euclidiana para auxiliar na identificação do contrato com a melhor relação de custo-benefício.

A Tabela 4 apresenta as particularidades existentes para cada um dos contratos SLAs adotados. Nessa tabela, assumindo a manutenção corretiva, pode-se observar que TTM representa o tempo total de manutenção (TTM + TA + MTTR) e envolve o tempo de deslocamento (TTD), o tempo de administrativo (TA) e o MTTR. Assumindo manutenção preventiva, outros tempos devem ser levados em consideração. Sendo assim, para a manutenção preventiva, o TTM é composto por TA, FreqMP (frequência da realização da manutenção preventiva) e MTTR. O TTD (tempo de deslocamento) considera o tempo médio gasto pelas equipes de manutenção para deslocar de seu local de trabalho até a empresa que solicitou o reparo; TA (tempo administrativo) corresponde ao tempo para notar a falha e solicitar o reparo; MTTR é o tempo médio para reparo. No caso da manutenção preventiva/corretiva também se utiliza a métrica FreqMP, que corresponde a frequência (periodicidade) da realização da manutenção preventiva.

Table 4. Contratos $S L A$

\begin{tabular}{lccccc}
\hline SLA & TTM & TTD & TA & MTTR & FreqMP \\
\hline I & $120 \mathrm{~h}$ & $18 \mathrm{~h}$ & $98 \mathrm{~h}$ & $4 \mathrm{~h}$ & $17520 \mathrm{~h}(2$ anos $)$ \\
II & $48 \mathrm{~h}$ & $10 \mathrm{~h}$ & $34 \mathrm{~h}$ & $4 \mathrm{~h}$ & $13140 \mathrm{~h}(1$ ano e meio $)$ \\
III & $24 \mathrm{~h}$ & $6 \mathrm{~h}$ & $14 \mathrm{~h}$ & $4 \mathrm{~h}$ & $8760 \mathrm{~h}(1$ anos $)$ \\
IV & $8 \mathrm{~h}$ & $3 \mathrm{~h}$ & $1 \mathrm{~h}$ & $4 \mathrm{~h}$ & $4380 \mathrm{~h}(6$ meses $)$ \\
\hline
\end{tabular}

O contrato de SLA para a manutenção corretiva considera as métricas: (i) o custo gasto com equipamento, este corresponde ao percentual gasto pelos equipamentos anualmente $\mathrm{x}$ custo de aquisição dos equipamentos; (ii) o custo da manutenção corretiva, corresponde ao custo de reparo dos equipamentos (30\% do valor de compra do equipamento); (iii) o custo com o time de manutenção, este custo pode variar em função da quantidade de times de manutenção disponíveis para fazer o reparo, sendo o custo para cada equipe de $\mathrm{R} \$ 2.500,00$. Para a manutenção preventiva, deve-se considerar também o custo extra da realização de uma manutenção periódica, valor obtido considerando um percentual de $25 \%$ do valor do custo gasto com equipamento. Vale ressaltar que os custos variam de acordo com o contrato de SLA adotado. A Tabela 2 apresenta o custo de aquisição de cada equipamento utilizado nesta análise e obtidos com base em [28] [29].

A Tabela 5 apresenta os contratos de $S L A$ para a manutenção corretiva. Conforme pode ser observado, foram criados os seguintes contratos: SLA 1, SLA 2, SLA 3 e SLA 4. O tempo de reparo total para cada contrato $S L A$ foi de 120 horas, 48 horas, 24 horas e 8 horas, respectivamente.

A Tabela 6 apresenta os contratos de $S L A$ para a manutenção preventiva. Existe, porém, um dado extra que é a frequência da manutenção preventiva, podendo ser realizada a cada: dois anos (17520 horas), um ano e meio (13140 horas), 1 anos ( 8760 horas) e 6 meses (4380), dependendo do contrato escolhido.

Vale salientar que o custo do contrato de SLA para as arquiteturas A4 e A5, em ambas as políticas de manutenção, apresentaram os mesmos valores. Isso ocorreu devido ao fato que o custo gasto para reparar os equipamentos power strip e STS serem relativamente baixos, para a arquitetura A4. Na arquitetura A5, tem-se a remoção do STS e a inserção de um novo power strip. Porém, o valor deste dispositivo levando em consideração o custo gasto para reparar esse equipamento é muito baixo. Logo, o custo não vai influenciar no valor dos contratos de SLAs das arquiteturas A4 e A5.

Vale ressaltar que para o caso da manutenção corretiva são avaliadas as arquiteturas (A1, ..., A5). Porém, para a manutenção preventiva, foram avaliadas apenas as arquiteturas $(\mathrm{A} 2, \ldots, \mathrm{A} 5)$. Isso ocorreu pelo fato desta política de 
Table 5. Contrato $S L A$ - Manutenção Corretiva

\begin{tabular}{|c|c|c|c|c|}
\hline \multicolumn{5}{|c|}{ Arquitetura A1 } \\
\hline$S L A$ & TTM(h) & Custo do Contrato $1 \mathrm{EQ}$ & Custo do Contrato $2 \mathrm{EQ}$ & Custo do Contrato $3 \mathrm{EQ}$ \\
\hline SLA I & $(24 * 5)=120 \mathrm{~h}$ & $\mathrm{R} \$ 7.632,29$ & $\mathrm{R} \$ 10.132,29$ & $\mathrm{R} \$ 12.632,29$ \\
\hline SLA II & $(24 * 2)=48 \mathrm{~h}$ & $\mathrm{R} \$ 7.750,73$ & $\mathrm{R} \$ 10.250,73$ & $\mathrm{R} \$ 12.750,73$ \\
\hline SLA III & 24 & $\mathrm{R} \$ 7.881,01$ & $\mathrm{R} \$ 10.381,01$ & $\mathrm{R} \$ 12.881,01$ \\
\hline SLA IV & $8 \mathrm{~h}$ & $\mathrm{R} \$ 8.024,32$ & $\mathrm{R} \$ 10.524,32$ & $\mathrm{R} \$ 13.024,32$ \\
\hline \multicolumn{5}{|c|}{ Arquitetura A2 } \\
\hline$S L A$ & TTM(h) & Custo do Contrato 1 EQ & Custo do Contrato 2 EQ & Custo do Contrato $3 \mathrm{EQ}$ \\
\hline SLA I & $(24 * 5)=120 \mathrm{~h}$ & $\mathrm{R} \$ 22.359,23$ & $\mathrm{R} \$ 24.859,23$ & $\mathrm{R} \$ 27.359,23$ \\
\hline SLA II & $(24 * 2)=48 \mathrm{~h}$ & $\mathrm{R} \$ 22.817,51$ & $\mathrm{R} \$ 25.317,51$ & $\mathrm{R} \$ 27.817,51$ \\
\hline SLA III & 24 & $\mathrm{R} \$ 23.321,63$ & $\mathrm{R} \$ 25.821,63$ & $\mathrm{R} \$ 28.321,63$ \\
\hline SLA IV & $8 \mathrm{~h}$ & $\mathrm{R} \$ 23.876,16$ & $\mathrm{R} \$ 26.376,16$ & $\mathrm{R} \$ 28.876,16$ \\
\hline \multicolumn{5}{|c|}{ Arquitetura A3 } \\
\hline$S L A$ & $\operatorname{TTM}(\mathrm{h})$ & Custo do Contrato $1 \mathrm{EQ}$ & Custo do Contrato 2 EQ & Custo do Contrato $3 \mathrm{EQ}$ \\
\hline$S L A \mathrm{I}$ & $(24 * 5)=120 \mathrm{~h}$ & $\mathrm{R} \$ 23.005,73$ & $\mathrm{R} \$ 25.505,73$ & $\mathrm{R} \$ 28.005,73$ \\
\hline SLA II & $(24 * 2)=48 \mathrm{~h}$ & $\mathrm{R} \$ 23.478,94$ & $\mathrm{R} \$ 25.978,94$ & $\mathrm{R} \$ 28.478,94$ \\
\hline SLA III & 24 & $\mathrm{R} \$ 23.999,47$ & $\mathrm{R} \$ 26.499,47$ & $\mathrm{R} \$ 28.999,47$ \\
\hline SLA IV & $8 \mathrm{~h}$ & $\mathrm{R} \$ 24.572,05$ & $\mathrm{R} \$ 27.072,05$ & $\mathrm{R} \$ 29.572,05$ \\
\hline \multicolumn{5}{|c|}{ Arquitetura A4 } \\
\hline$S L A$ & TTM(h) & Custo do Contrato 1 EQ & Custo do Contrato 2 EQ & Custo do Contrato $3 \mathrm{EQ}$ \\
\hline$S L A \mathrm{I}$ & $(24 * 5)=120 \mathrm{~h}$ & $\mathrm{R} \$ 23.029,17$ & $\mathrm{R} \$ 25.529,17$ & $\mathrm{R} \$ 28.029,17$ \\
\hline SLA II & $(24 * 2)=48 \mathrm{~h}$ & $\mathrm{R} \$ 23.739,80$ & $\mathrm{R} \$ 26.239,80$ & $\mathrm{R} \$ 28.739,80$ \\
\hline SLA III & 24 & $\mathrm{R} \$ 24.557,02$ & $\mathrm{R} \$ 27.057,02$ & $\mathrm{R} \$ 29.557,02$ \\
\hline SLA IV & $8 \mathrm{~h}$ & $\mathrm{R} \$ 25.496,82$ & $\mathrm{R} \$ 27.996,82$ & $\mathrm{R} \$ 30.496,82$ \\
\hline \multicolumn{5}{|c|}{ Arquitetura A5 } \\
\hline$S L A$ & $\operatorname{TTM}(\mathrm{h})$ & Custo do Contrato 1 EQ & Custo do Contrato 2 EQ & Custo do Contrato $3 \mathrm{EQ}$ \\
\hline SLA I & $(24 * 5)=120 \mathrm{~h}$ & $\mathrm{R} \$ 23.029,17$ & $\mathrm{R} \$ 25.529,17$ & $\mathrm{R} \$ 28.029,17$ \\
\hline SLA II & $(24 * 2)=48 \mathrm{~h}$ & $\mathrm{R} \$ 23.739,80$ & $\mathrm{R} \$ 26.239,80$ & $\mathrm{R} \$ 28.739,80$ \\
\hline SLA III & 24 & $\mathrm{R} \$ 24.557,02$ & $\mathrm{R} \$ 27.057,02$ & $\mathrm{R} \$ 29.557,02$ \\
\hline SLA IV & $8 \mathrm{~h}$ & $\mathrm{R} \$ 25.496,82$ & $\mathrm{R} \$ 27.996,82$ & $\mathrm{R} \$ 30.496,82$ \\
\hline
\end{tabular}

manutenção ser utilizada apenas em equipamentos redundantes, cenário observado a partir da arquitetura A2.

As Tabelas 7 e 8 apresentam respectivamente os resultados da disponibilidade da manutenção corretiva e preventiva, obtidos para cada arquitetura levando em consideração os diferentes SLAs e considerando diferentes números de equipes de manutenção disponíveis.

A arquitetura A4 foi escolhida para ser analisada, pois entre as arquiteturas apresentadas nas Tabelas 7 e 8, ela é a que apresenta a melhor relação custo-benefício. Conforme pode ser observado nas Tabelas 7 e 8 , o contrato $S L A$ do nível 4 oferece uma maior disponibilidade do que os demais contratos. No caso da manutenção corretiva, tem-se a disponibilidade (números de noves) para o contrato de nível 1 e 4 de 4,47 e 5,68, respectivamente. No caso da manutenção preventiva, tem-se, respectivamente para o contrato de nível 1 e 4 a disponibilidade de 4,77 e 5,94.

As Tabelas 7 e 8 mostram que a manutenção preventiva apresenta melhores resultados para a disponibilidade quando comparado a manutenção corretiva.

Vale ressaltar que o comportamento dos resultados obtidos para a arquitetura A4 se repete para as arquiteturas A2 e A3, onde os resultados obtidos pela manutenção preventiva apresentam melhores resultados. Porém, para a arquitetura A5, observou-se uma particularidade nos contratos $S L A 3$ e SLA4, onde ocorre manutenções preventivas com intervalos de um ano (8760h) e seis meses (4380h), a disponibilidade da manutenção preventiva apresenta valores menores que o cenário levando em consideração somente a manutenção corretiva. Porém, para os $S L A 1$ e $S L A 2$, com a manutenção ocorrendo em um intervalo de dois anos $(17520 \mathrm{~h})$ e um ano e meio (13140h), obteve-se melhores resultados. 
Evaluating the impact of maintenance policies associated to SLA contracts

Table 6. Contato $S L A$ - Manutenção Preventiva

\begin{tabular}{|c|c|c|c|c|c|}
\hline \multicolumn{6}{|c|}{ Arquitetura A2 } \\
\hline$S L A$ & FreqMP & TTM(h) & Custo do Contrato $1 \mathrm{EQ}$ & Custo do Contrato $2 \mathrm{EQ}$ & Custo do Contrato 3 EQ \\
\hline$S L A \mathrm{I}$ & $(2$ anos $)=26280$ & $(24 * 5)=120 \mathrm{~h}$ & $\mathrm{R} \$ 26.178,31$ & $\mathrm{R} \$ 28.678,31$ & $\mathrm{R} \$ 31.178,3$ \\
\hline SLA II & $(1.5)=17520$ & $(24 * 2)=48 \mathrm{~h}$ & $\mathrm{R} \$ 27.018,50$ & $\mathrm{R} \$ 29.518,50$ & $\mathrm{R} \$ 32.018,50$ \\
\hline SLA III & $(1$ anos $)=8760$ & $24 \mathrm{~h}$ & $\mathrm{R} \$ 27.942,72$ & $\mathrm{R} \$ 30.442,72$ & $\mathrm{R} \$ 32.942,72$ \\
\hline SLA IV & $(6$ meses $)=4380$ & $8 \mathrm{~h}$ & $\mathrm{R} \$ 28.959,36$ & $\mathrm{R} \$ 31.459,36$ & $\mathrm{R} \$ 33.959,36$ \\
\hline \multicolumn{6}{|c|}{ Arquitetura A3 } \\
\hline$S L A$ & FreqMP & $\operatorname{TTM}(\mathrm{h})$ & Custo do Contrato $1 \mathrm{EQ}$ & Custo do Contrato 2 EQ & Custo do Contrato 3 \\
\hline SLA I & $(2$ anos $)=26280$ & $(24 * 5)=120 \mathrm{~h}$ & $\mathrm{R} \$ 26.949,14$ & $\mathrm{R} \$ 29.449,14$ & $\mathrm{R} \$ 31.949,14$ \\
\hline SLA II & $(1.5)=17520$ & $(24 * 2)=48 \mathrm{~h}$ & $\mathrm{R} \$ 27.816,69$ & $\mathrm{R} \$ 30.316,69$ & $\mathrm{R} \$ 32.816,69$ \\
\hline SLA III & $(1$ anos $)=8760$ & $24 \mathrm{~h}$ & $\mathrm{R} \$ 28.770,99$ & $\mathrm{R} \$ 31.270,99$ & $\mathrm{R} \$ 33.770,99$ \\
\hline SLA IV & $(6$ meses $)=4380$ & $8 \mathrm{~h}$ & $\mathrm{R} \$ 29.820,73$ & $\mathrm{R} \$ 32.320,73$ & $\mathrm{R} \$ 34.820,73$ \\
\hline \multicolumn{6}{|c|}{ Arquitetura A4 } \\
\hline$S L A$ & FreqMP & $\operatorname{TTM}(\mathrm{h})$ & Custo do Contrato $1 \mathrm{EQ}$ & Custo do Contrato 2 EQ & Custo do Contrato $3 \mathrm{EQ}$ \\
\hline SLA I & $(2$ anos $)=26280$ & $(24 * 5)=120 \mathrm{~h}$ & $\mathrm{R} \$ 26.977,09$ & $\mathrm{R} \$ 29.477,09$ & $\mathrm{R} \$ 31.977,09$ \\
\hline SLA II & $(1.5)=17520$ & $(24 * 2)=48 \mathrm{~h}$ & $\mathrm{R} \$ 30.451,26$ & $\mathrm{R} \$ 30.345,63$ & $\mathrm{R} \$ 32.845,63$ \\
\hline SLA III & $(1$ anos $)=8760$ & $24 \mathrm{~h}$ & $\mathrm{R} \$ 31.667,22$ & $\mathrm{R} \$ 31.301,03$ & $\mathrm{R} \$ 33.801,03$ \\
\hline SLA IV & $(6$ meses $)=4380$ & $8 \mathrm{~h}$ & $\mathrm{R} \$ 33.004,77$ & $\mathrm{R} \$ 32.351,96$ & $\mathrm{R} \$ 34.851,96$ \\
\hline \multicolumn{6}{|c|}{ Arquitetura A5 } \\
\hline$S L A$ & FreqMP & $\mathrm{TTM}(\mathrm{h})$ & Custo do Contrato $1 \mathrm{EQ}$ & Custo do Contrato $2 \mathrm{EQ}$ & Custo do Contrato $3 \mathrm{EQ}$ \\
\hline$S L A$ I & $(2$ anos $)=26280$ & $(24 * 5)=120 \mathrm{~h}$ & $\mathrm{R} \$ 26.977,09$ & $\mathrm{R} \$ 29.477,09$ & $\mathrm{R} \$ 31.977,09$ \\
\hline SLA II & $(1.5)=17520$ & $(24 * 2)=48 \mathrm{~h}$ & $\mathrm{R} \$ 30.451,26$ & $\mathrm{R} \$ 30.345,63$ & $\mathrm{R} \$ 32.845,63$ \\
\hline SLA III & $(1$ anos $)=8760$ & $24 \mathrm{~h}$ & $\mathrm{R} \$ 31.667,22$ & $\mathrm{R} \$ 31.301,03$ & $\mathrm{R} \$ 33.801,03$ \\
\hline SLA IV & $(6$ meses $)=4380$ & $8 \mathrm{~h}$ & $\mathrm{R} \$ 33.004,77$ & $\mathrm{R} \$ 32.351,96$ & $\mathrm{R} \$ 34.851,96$ \\
\hline
\end{tabular}

Legenda: FreqMP - Frequência na realização da manutenção preventiva; TTM - Tempo total de manutenção; EQ - Equipe de manutenção;

A manutenção preventiva permite que os equipamentos voltem a funcionar em estado de novo (sem ter o MTTF degradado pela ação do tempo). Já na manutenção corretiva, com o passar do tempo, os equipamentos passam a falhar com mais frequência (MTTF degradado). Os resultados obtidos mostram que o uso de uma manutenção preventiva é de grande valia, pois permite que o sistema tenha uma disponibilidade melhor quando comparada unicamente com a manutenção corretiva.

Foi avaliado também o impacto da utilização de contratos com mais equipes de manutenção no sistema, tendo assim, análises referentes a uma, duas e três equipes de manutenção no sistema. É possível observar que a utilização de mais equipes de manutenção aumenta a disponibilidade do sistema. Porém, para ambas políticas de manutenção, a utilização de duas equipes são suficientes para obter a maior disponibilidade do sistema, visto que não tem variação da disponibilidade com a utilização de três equipes de manutenção.

Avaliando o custo dos contatos de $S L A$ associado as políticas de manutenção (Tabelas 5 e 6), percebe-se que para cada nível do contrato, os preços dos contratos tendem a ficar mais caro. Porém, mesmo existindo o aumento no valor do contrato, anal- isando as Tabelas 7 e 8, a disponibilidade para cada nível do contrato também tende a crescer.

Este artigo utiliza a distância Euclidiana para se detectar a melhor relação custo-benefício. As Tabelas 9 e 10 apresentam as distâncias euclidianas para as políticas de manutenção e associada a cada contrato de $S L A$, considerando o downtime e custo do contrato. Vale salientar que os resultados apresentados consideram o eixo " $\mathrm{X}$ " como sendo os valores obtidos para o downtime, vinculado a cada contrato e o eixo "Y" como sendo o custo de cada contrato de $S L A$. A distância euclidiana foi obtida através da fórmula: $D=\sqrt{\left(X_{f}-X_{i}\right)^{2}+\left(Y_{f}-Y_{i}\right)^{2}}$; onde $X_{i}$ ou $Y_{i}$ é a distância inicial e $X_{f}$ ou $Y_{f}$ é a distância final. Como as métricas citadas apresentam ordem de grandeza distintas, os resultados são apresentados como valores absolutos e normalizados em relação ao componente de maior valor, através da equação: $\ln _{i}=\frac{I_{i}}{I_{x}}$, sendo $\ln _{i}$ o índice normalizado para o componente i; $I_{i}$ o valor não normalizado para o componente $\mathrm{i}$; e $I_{x}$ corresponde ao maior índice não normalizado entre os componentes.

Assumindo os resultados das distâncias euclidianas para ambas políticas de manutenção, pode-se identificar a de menor valor que é 0,8259 para o $S L A 3$ com um time de manutenção 
Evaluating the impact of maintenance policies associated to SLA contracts

Table 7. Disponibilidade (9's) da Manutenção Corretiva

\begin{tabular}{|c|c|c|c|}
\hline \multicolumn{4}{|c|}{ Arquitetura A1 } \\
\hline$S L A$ & Disp.(9's)1equipe & Disp.(9's)2equipes & Disp.(9's)3equipes \\
\hline SLA 1 & $0,99869237(2,88)$ & $0,99869274(2,88)$ & $0,99869274(2,88)$ \\
\hline SLA 2 & $0,99947671(3,28)$ & $0,99947677(3,28)$ & $0,99947677(3,28)$ \\
\hline SLA 3 & $0,99973832(3,58)$ & $0,99973833(3,58)$ & $0,99973833(3,58)$ \\
\hline SLA 4 & $0,99991276(4,06)$ & $0,99991277(4,06)$ & $0,99991277(4,06)$ \\
\hline \multicolumn{4}{|c|}{$\begin{array}{l}\text { Arquitetura A2 } \\
\end{array}$} \\
\hline$S L A$ & Disp.(9's)1equipe & Disp.(9's)2equipes & Disp.(9's)3equipes \\
\hline SLA 1 & $0,99963894(3,44)$ & $0,99964058(3,44)$ & $0,99964058(3,44)$ \\
\hline SLA 2 & $0,99985617(3,84)$ & $0,99985643(3,84)$ & $0,99985643(3,84)$ \\
\hline SLA 3 & $0,99992818(4,14)$ & $0,99992825(4,14)$ & $0,99992825(4,14)$ \\
\hline SLA 4 & $0,99997608(4,62)$ & $0,99997609(4,62)$ & $0,99997609(4,62)$ \\
\hline \multicolumn{4}{|c|}{ Arquitetura A3 } \\
\hline$S L A$ & Disp.(9's)1 lequipe & Disp.(9's)2equipes & Disp.(9's)3equipes \\
\hline SLA 1 & $0,99980832(3,72)$ & $0,99981003(3,7$ & \\
\hline SLA 2 & $0,99992404(4,12)$ & $0,99992431(4,12)$ & $0,99992431(4,12)$ \\
\hline SLA 3 & $0,99996214(4,42)$ & $0,9999622(4,42)$ & $0,9999622(4,42)$ \\
\hline SLA 4 & $0,99998741(4,90)$ & $0,99998741(4,90)$ & $0,99998741(4,90)$ \\
\hline \multicolumn{4}{|c|}{ Arquitetura A4 } \\
\hline$S L A$ & Disp.(9's)1 lequipe & Disp.(9's)2equipes & Disp.(9's)3equipes \\
\hline SLA 1 & $0,99996577(4,47)$ & $0,99996751(4,49)$ & $0,99996751(4,49)$ \\
\hline SLA 2 & $0,99998712(4,89)$ & $0,9999874(4,90)$ & $0,9999874(4,90)$ \\
\hline SLA 3 & $0,9999937(5,20)$ & $0,99999377(5,21)$ & $0,99999377(5,21)$ \\
\hline SLA 4 & $0,99999793(5,68)$ & $0,99999794(5,69)$ & $0,99999794(5,69)$ \\
\hline \multicolumn{4}{|c|}{ Arquitetura A5 } \\
\hline$S L A$ & Disp.(9's)1equipe & Disp.(9's)2equipes & Disp.(9's)3equipes \\
\hline SLA 1 & $0,99999658(5,5)$ & $0,99999829(5,8)$ & $0,99999829(5,8)$ \\
\hline SLA 2 & $0,99999945(6,3)$ & $0,99999973(6,6)$ & $0,99999973(6,6)$ \\
\hline SLA 3 & $0,99999986(6,9)$ & $0,99999993(7,2)$ & $0,99999993(7,2)$ \\
\hline SLA 4 & $0,99999998(7,7)$ & $0,99999999(8,0)$ & $0,99999999(8,0)$ \\
\hline
\end{tabular}

corretiva. Vale ressaltar que o contrato do nível três com apenas uma equipe para a manutenção preventiva apresenta resultados bem próximos com uma distância de 0,8481 .

A Figura 11 apresenta os resultados da manutenção corretiva da arquietura A4, para todos os contratos SLAs, levando em consideração uma, duas e três equipes de manutenção. Similarmente, a Figure 12 apresenta os resultados para a manutenção preventiva. Observa-se que entre os contratos SLAs da manutenção corretiva, o contrato de SLA do nível três com apenas uma equipe de manutenção apresenta a menor distância euclidiana, sendo o mais indicado para ser utilizado. Similar resultado foi detectado na manutenção preventiva, onde o SLA3 com uma equipe de manutenção tem a menor distância Euclidiana. Para ambos os casos, esses contratos são apresentados como sendo os melhores, pois apresentam a menor distância euclidiana.

Analisando os contratos destinados a arquitetura A4, o os meninos tão brincando $S L A 1$ com três equipes de manutenção é considerado o contrato menos indicado para ser utilizado, pois apresenta a maior distância euclidiana para a manutenção corretiva. Realizando a mesma comparação para a manutenção preventiva, verificou-se que o contrato de $S L A 1$ com três equipes de manutenção é o menos indicado para ser utilizado. Vale ressaltar que esses contratos apontados como os menos indicados a serem utilizados, pois apresentam a maior distância
Table 8. Disponibilidade (9's) da Manutenção Preventiva

\begin{tabular}{llll}
\hline \multicolumn{4}{c}{ Arquitetura A2 } \\
\hline SLA & Disp.(9's)1equipe & Disp.(9's)2equipes & Disp.(9's)3equipes \\
\hline SLA 1 & $0,9997951(3,69)$ & $0,99979567(3,69)$ & $0,99979567(3,69)$ \\
SLA 2 & $0,99992097(4,10)$ & $0,99992106(4,10)$ & $0,99992106(4,10)$ \\
SLA 3 & $0,99996182(4,42)$ & $0,99996185(4,42)$ & $0,99996185(4,42)$ \\
SLA 4 & $0,99998765(4,91)$ & $0,99998767(4,91)$ & $0,99998767(4,91)$ \\
\hline \multicolumn{4}{c}{ Arquitetura A3 } \\
\hline SLA & Disp.(9's)1equipe & Disp.(9's)2equipes & Disp.(9's)3equipes \\
\hline SLA 1 & $0,99989878(4,00)$ & $0,99989928(4,00)$ & $0,99989928(4,00)$ \\
SLA 2 & $0,99996036(4,40)$ & $0,99996044(4,40)$ & $0,99996044(4,40)$ \\
SLA 3 & $0,9999805(4,71)$ & $0,99998052(4,71)$ & $0,99998052(4,71)$ \\
SLA 4 & $0,99999354(5,19)$ & $0,99999357(5,19)$ & $0,99999357(5,19)$ \\
\hline \multicolumn{5}{c}{ Arquitetura A4 } \\
\hline SLA & Disp.(9's)1equipe & Disp.(9's)2equipes & Disp.(9's)3equipes \\
\hline SLA 1 & $0,999983(4,77)$ & $0,99998348(4,78)$ & $0,99998348(4,78)$ \\
SLA 2 & $0,99999347(5,19)$ & $0,99999354(5,19)$ & $0,99999354(5,19)$ \\
SLA 3 & $0,99999676(5,49)$ & $0,99999679(5,49)$ & $0,99999679(5,49)$ \\
SLA 4 & $0,99999884(5,94)$ & $0,99999888(5,95)$ & $0,99999888(5,95)$ \\
\hline \multicolumn{5}{c}{ Arquitetura A5 } \\
\hline SLA & Disp.(9's)1equipe & Disp.(9's)2equipes & Disp.(9's)3equipes \\
\hline SLA 1 & $0,99999905(6,02)$ & $0,99999951(6,31)$ & $0,99999951(6,31)$ \\
SLA 2 & $0,9999998(6,70)$ & $0,99999988(6,92)$ & $0,99999988(6,92)$ \\
SLA 3 & $0,99999989(6,96)$ & $0,99999991(7,05)$ & $0,99999991(7,05)$ \\
SLA 4 & $0,99999984(6,80)$ & $0,99999989(6,96)$ & $0,99999989(6,96)$ \\
\hline
\end{tabular}

Table 9. Distância Euclidiana - Manutenção Corretiva

\begin{tabular}{clll}
\hline SLA & 1 Equipe & 2 Equipes & 3 Equipes \\
\hline SLA 1 & 1,253086821 & 1,265571269 & 1,321225187 \\
SLA 2 & 0,864607817 & 0,935843672 & 1,011725802 \\
SLA 3 & 0,825998113 & 0,905683923 & 0,986124981 \\
SLA 4 & 0,838232711 & 0,919994714 & 1,001809247 \\
\hline
\end{tabular}

Table 10. Distância Euclidiana - Manutenção Preventiva

\begin{tabular}{clll}
\hline SLA & 1 Equipe & 2 Equipes & 3 Equipes \\
\hline SLA1 & 1,264575150 & 1,288281963 & 1,336471037 \\
SLA2 & 0,886508641 & 0,950010479 & 1,016159224 \\
SLA3 & 0,848074862 & 0,917748831 & 0,988056358 \\
SLA4 & 0,859249704 & 0,930603034 & 1,002167892 \\
\hline
\end{tabular}

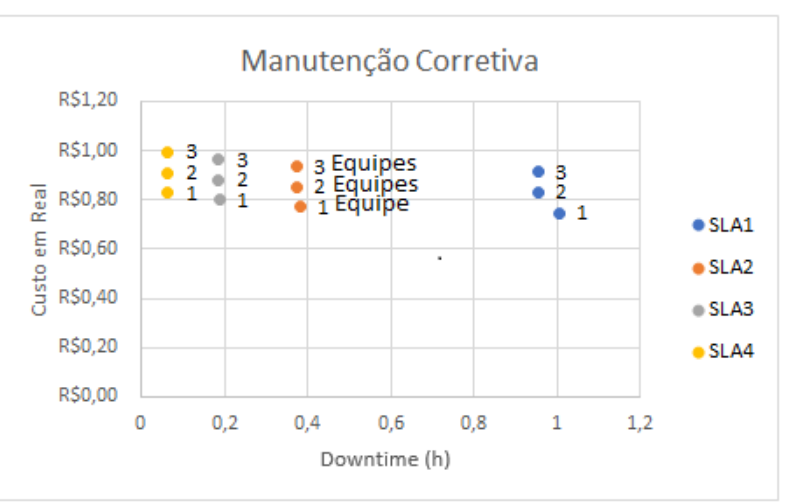

Figure 11. Downtime e Custo da Manutenção Corretiva Considerando a Arquitetura A4 


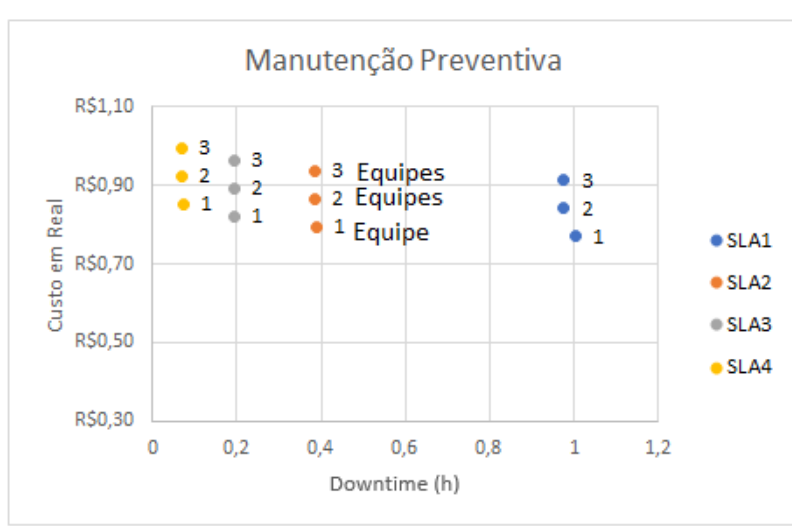

Figure 12. Downtime e Custo da Manutenção Preventiva Considerando a Arquitetura A4

euclidiana, logo possuem o maiordowntime e/ou custo entre os contratos analisados para a arquitetura A4.

\section{Conclusão}

Este trabalho apresentou uma abordagem de modelagem que considera as vantagens das redes de Petri (SPN) para analisar o impacto das políticas de manutenção na disponibilidade vinculadas a contratos SLA de sistemas data center. Experimentos evidenciaram a viabilidade da abordagem proposta, onde modelos SPN propostos fornecem resultados importantes para auxiliar os projetista de data center na escolha da melhor política de manutenção. Conforme observado no estudo de caso, a utilização da manutenção preventiva foi de grande valia, pois os resultados obtidos evidenciaram que a disponibilidade do sistema foi superior ao se utilizar esse tipo de abordagem. Porém, os custos dos contratos também foram superiores. Fazendo uso da distância euclidiana avaliando o downtime e custo, observou-se que é possível se detectar os melhores contratos levando em consideração tais métricas conflitantes.

Como trabalho futuro, estaremos ampliando a metodologia proposta para avaliar outras infraestruturas de data center (TI e refrigeração) e, também, avaliando a utilização de métodos de otimização.

\section{Agradecimentos}

Os autores gostariam de agradecer à FACEPE e ao CNPq pelo suporte financeiro a esta pesquisa.

\section{Contribuição dos autores}

Os autores tiveram contribuições similares neste artigo.

\section{References}

[1] BORKOWSKI, M.; HANS, P. Reliability centered maintenance (rcm) handbook. Naval Sea Systems Command, US, 2007.
[2] WERNKE, R. Gestão de custos: uma abordagem prática. são paulo: Atlas, 2001. _. Gestão de custos: uma abordagem prática, v. 2, 2016.

[3] VERDI, F. L. et al. Novas arquiteturas de data center para cloud computing. Minicursos do XXVIII SBRC, p. 103-152, 2010.

[4] CALLOU, G. et al. Impact analysis of maintenance policies on data center power infrastructure. In: IEEE. Systems Man and Cybernetics (SMC), 2010 IEEE International Conference on. [S.1.], 2010. p. 526-533.

[5] SILVA, B. et al. Astro: An integrated environment for dependability and sustainability evaluation. Sustainable computing: informatics and systems, Elsevier, v. 3, n. 1, p. 1-17, 2013.

[6] SILVA, B. et al. Geoclouds modes: A perfomability evaluation tool for disaster tolerant iaas clouds. In: IEEE. Systems Conference (SysCon), 2014 8th Annual IEEE. [S.1.], 2014. p. 116-122.

[7] ADDABBO, T. et al. High performance liquid chromatography lcc analysis. In: IEEE. Instrumentation and Measurement Technology Conference (I2MTC), 2015 IEEE International. [S.1.], 2015. p. 945-950.

[8] SAID, U.; TAGHIPOUR, S. Modeling failure and maintenance effects of a system subject to multiple preventive maintenance types. In: IEEE. Reliability and Maintainability Symposium (RAMS), 2016 Annual. [S.1.], 2016. p. 1-7.

[9] WANG, W. et al. On multi-state system with intervalvalued states under preventive maintenance and minimal repairs. In: IEEE. Reliability Systems Engineering (ICRSE), 2017 Second International Conference on. [S.1.], 2017. p. 17.

[10] DINIZ, M. et al. Quantificando a confiabilidade de redes elétricas: Uma abordagem via modelos markovianos com recompensas.

[11] BICHPURIYA, Y. K.; NAVALKAR, P. V.; SOMAN, S. Benchmarking of reliability indices for electricity distribution utilities: approach and discussion. IET, 2011.

[12] LIU, Y.; TRIVEDI, K. S. Survivability quantification: The analytical modeling approach. International Journal of Performability Engineering, v. 2, n. 1, p. 29-44, 2006.

[13] VIANA, H. R. G. PCM-Planejamento e Controle da Manutenção. [S.1.]: Qualitymark Editora Ltda, 2002.

[14] ALMEIDA, A. T. d. Modelagem multicritério para seleção de intervalos de manutenção preventiva baseada na teoria da utilidade multiatributo. Pesquisa Operacional, SciELO Brasil, v. 25, n. 1, p. 69-81, 2005.

[15] GUIMARÃES, L. M.; NOGUEIRA, C. F.; SILVA, M. D. B. da. Manutenção industrial: implementação da manutenção produtiva total (tpm). e-xacta, v. 5, n. 1, 2012.

[16] OTANI, M.; MACHADO, W. V. A proposta de desenvolvimento de gestão da manutenção industrial na busca da 
excelência ou classe mundial. Revista Gestão Industrial, v. 4, n. 2, 2008.

[17] RIBEIRO, J. B.; GOMES, M. C. Manutenção preditiva em motores de combustão interna por análise do óleo lubrificante. Arruda, Alberto Luiz de Luna, 2016.

[18] BOTELHO, D. C. d. C.; CABRAL, V. R. Estudo do impacto do desalinhamento de máquina rotativas no consumo de energia elétrica. Renato Couto de Almeida, 2017.

[19] LUCATELLI, M. V. et al. Proposta de aplicação da manutenção centrada em confiabilidade em equipamentos médico-hospitalares. Florianópolis, SC, 2002.

[20] JAIN, R. K. Art of Computer Systems Performance Analysis: Techniques for Experimental Design Measurements... Simulation and Modeling. [S.1.]: John Wiley, 2015.

[21] SOUSA, E. T. G. d. Modelagem de desempenho, dependabilidade e custo para o planejamento de infraestruturas de nuvens privadas. UNIVERSIDADE FEDERAL DE PERNAMBUCO, 2015.

[22] ALPENDRE, M. Service level agreement: Um conceito a saber usar. Departamento de Engenharia Informtica, Universidade de Coimbra, 2006.
[23] STURM, R.; MORRIS, W.; JANDER, M. Foundations of service level management. [S.I.]: Sams Indianapolis, IN, 2000. v. 13.

[24] HILES, A. Service-Level Agreements in Business Continuity Management. 2004.

[25] LUDWIG, H. et al. Web service level agreement (wsla) language specification. Ibm corporation, p. 815-824, 2003.

[26] ZIMMERMANN, A. Modeling and evaluation of stochastic petri nets with timenet 4.1. In: IEEE. Performance evaluation methodologies and tools (VALUETOOLS), 2012 6th international conference on. [S.1.], 2012. p. 54-63.

[27] AVELAR, V. Comparing availability of various rack power redundancy configurations. APC White Paper, v. 48, p. 1-22, 2003.

[28] AMAZON. Amazon. https://www.amazon.com. Acesso em, 2018.

[29] RESTOCKIT. Restockit. http://www.restockit.com. Acesso em, 2018. 